\title{
Web Services in Multimedia Communication
}

\author{
Mohamed Hamdi, ${ }^{1}$ Nabil Tabbane, ${ }^{2}$ Tai-Hoon Kim, ${ }^{3}$ and Sajid Hussain ${ }^{4}$ \\ ${ }^{1}$ Computer Science and Networks Department, School of Communication Engineering (Sup'Com), University of Carthage, \\ Technopark El Ghazala, 2083 El Ghazala, Tunisia \\ ${ }^{2}$ Multimedia Mobile Radio Networks Research Unit (MEDIATRON), Sup'Com, University of Carthage, Technopark El Ghazala, \\ 2083 El Ghazala, Tunisia \\ ${ }^{3}$ Global Vision School, 101-ho, Jeongseong-vill, 593-7, Wonseong-dong, Dongnam-gu, Cheonan-si, Chungnam-do, Republic of Korea \\ ${ }^{4}$ Department of Business Administration, Fisk University, 321 Park Johnson Hall (PJ), Nashville, TN, USA
}

Correspondence should be addressed to Mohamed Hamdi, mmh@supcom.rnu.tn

Received 29 November 2012; Accepted 29 November 2012

Copyright (C) 2012 Mohamed Hamdi et al. This is an open access article distributed under the Creative Commons Attribution License, which permits unrestricted use, distribution, and reproduction in any medium, provided the original work is properly cited.

Multimedia communication has made a tremendous impact on web technologies and has been the basis for various applications ranging from videoconferencing to medical imaging and target surveillance. The advent of new efficient multimedia coding schemes has made it technologically feasible and economically viable to develop web services that encompass the real-time transmission of high-resolution multimedia content. For instance, Youtube users spend 3 billion hours per month on the site. Moreover, movies streamed by Netflix can reach 20 percent of US broadband traffic. This is just the beginning of a video flood that will swamp the web.

Multimedia communication and web services constitute the confluence point where the interest of service providers, telecommunication operators, and end users meet. Many digital services, such as pay-TV, confidential video conferencing, medical and military imaging systems, require reliable security in storage and transmission of digital images/videos. As the rapid progress of Internet in the digital world today, the security of digital images/videos has become increasingly important. In recent years, more and more consumer electronic services and devices, such as mobile phones and PDA (personal digital assistant), have also started to provide additional functions of saving and exchanging multimedia messages. The prevalence of multimedia technology in our society has promoted digital images and videos to play a more significant role than the traditional dull texts, which demands a serious protection of users' privacy. Because online videos are useful for a wide spectrum of web services, companies are reorganizing their software and network infrastructures to accommodate it. That focus has touched off major challenges over protocols, algorithms, and standards allowing the provision of reliable and high-quality performance in multimedia communication.

The objective of this special issue is to bring together research contributions on the design, specification, and implementation of architectures, protocols, and algorithms for current and future web services based on multimedia communication. The special issue is devoted to those areas of web technologies where real-time video and image transmission bring new insights to yield effective solutions for the problems of interest. The issue contains five highquality papers whose topics range from image encryption to automatic service discovery offering innovative methodologies, algorithms, and theoretical results by using the existing achievements in multimedia communication and by extending them to fit the specific needs of web applications.

This special issue covers a variety of recent research articles and comprehensive materials on recent technological advances in the field of Web Services for Multimedia Communication. We invited authors to submit original research articles that held with recent advances in Web services technology with application to multimedia communication. We received 8 submissions from authors around the world. Papers that passed our preliminary screening were passed to a rigorous review process involving experts in this field. Finally, five articles were accepted for this issue.

In the paper, "Image encryption using a lightweight stream encryption algorithm" by S. Bahrami and M. Naderi, a simple and lightweight stream encryption algorithm for image data 
is proposed. This algorithm operates on sections of the image using a different key for every section. The secret key of the encryption scheme is protected by a block cipher algorithm (such as AES). The authors discuss the robustness of the lightweight encryption scheme to meet-in-the-middle and chosen ciphertext attacks. A series of statistical tests have also been performed to give an idea on the protection level provided by the proposed encryption technique. For instance, the histogram analysis shows that the distribution of pixel brightness in the encrypted image is uniform. The speed of the encryption functions has also been shown to be convenient for real-time transmission.

The paper, "A novel k-out-of- $n$ oblivious transfer protocol from bilinear pairing" by J.-S. Chou, provides an oblivious transfer protocol that can be used in various applications like the signature of fair contracts, oblivious database searches, mental poker games, privacy-preserving auctions, and secure multiparty computations. In addition to cryptographic robustness, the author addresses the complexity of the protocol, which is essential for commercial applications. The major advantage of the proposed approach is that it can run without the secure channel required by the existing techniques to enforce protection against replay, denial of service, and man-in-the-middle attacks. The correctness of the scheme has been formally proved in addition to mutual authentication, privacy, and anti-replay properties.

In the paper, "Parlay $X$ Web services for policy and charging control in multimedia networks" by I. Atanasov, E. Pencheva, and D. Marinska, the authors investigate the capabilities of Parlay X Web services for Policy and Charging Control (PCC) in managing all Internet-protocol-based multimedia networks. They explore the requirements for open access to policy and charging control and assess the potential brought by Parlay X Web services compared to PCC. They propose an enhancement to Parlay X Web services to support PCC. The proposed improvement is articulated around the development of new interfaces for usage monitoring, the enhancement of the Quality of Service (QoS) interface, the development of new interfaces for access to QoS-related user data, and the enhancement of call notification functionality. The advantages of these enhancements have been highlighted through the description of case studies.

In the paper, "Seamless integration of RESTful services into the web of data", M. Lanthaler and C. Gütl study the seamless integration of RESTful services into semantic web technologies. The proposed idea allows data integration on an unprecedented scale. A new approach (called SEREDASj) is introduced to create machine-readable descriptions for RESTful services as a first step toward solving web development problems. It enables web developers to use tools and knowledge they are already familiar with. In fact, it does not require changes on the described Web services, it provides a viable upgrade path for existing infrastructure. Two algorithms have been proposed to translate SPARQ Update operations to HTTP requests interacting with a SEREDASjdescribed API. Auch standardized interface increases the developer's productivity and improves code readability.

In the paper, "A framework for automatic web service discovery based on semantics and NLP techniques", A. Adala,
N. Tabbane, and S. Tabbane propose a combination of semantic and NLP search techniques. This framework is based on natural language processing techniques to match a user request, expressed in natural language, with a semantic web services description. An efficient semantic matching technique has also been proposed to compute the semantic distance between ontological concepts. Compared to existing techniques, the proposed approach exhibits multiple advantages. It offers a simple syntax in terms of a list of keyword phrases and open vocabularies. It maps natural language words into ontological concepts. Furthermore, it does not make any assumption about the description language of the Web service. In fact, it integrates a mapping module which converts English terms from the WordNet database to Suggested Upper Merged Ontology (SUMO).

We hope that this issue will serve as a valuable reference and trend indicator for researchers and engineers in both industry and academia.

\section{Acknowledgments}

In closing, we would like to thank all authors for their valuable contributions to this feature topic. We are also grateful to the experts who participated in the review process and completed their reviews within a very tight schedule.

Mohamed Hamdi Nabil Tabbane Tai-Hoon Kim Sajid Hussain 

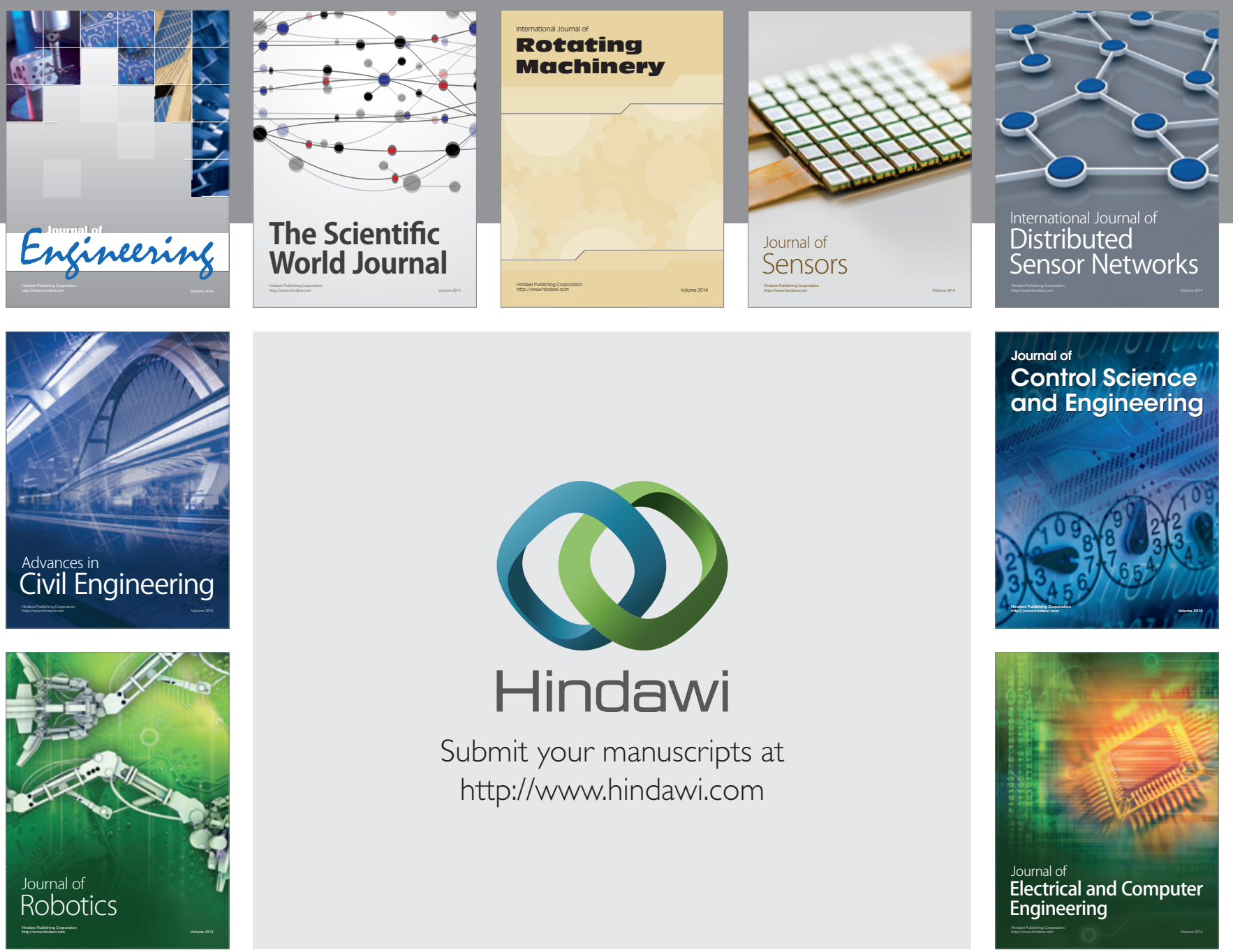

Submit your manuscripts at

http://www.hindawi.com
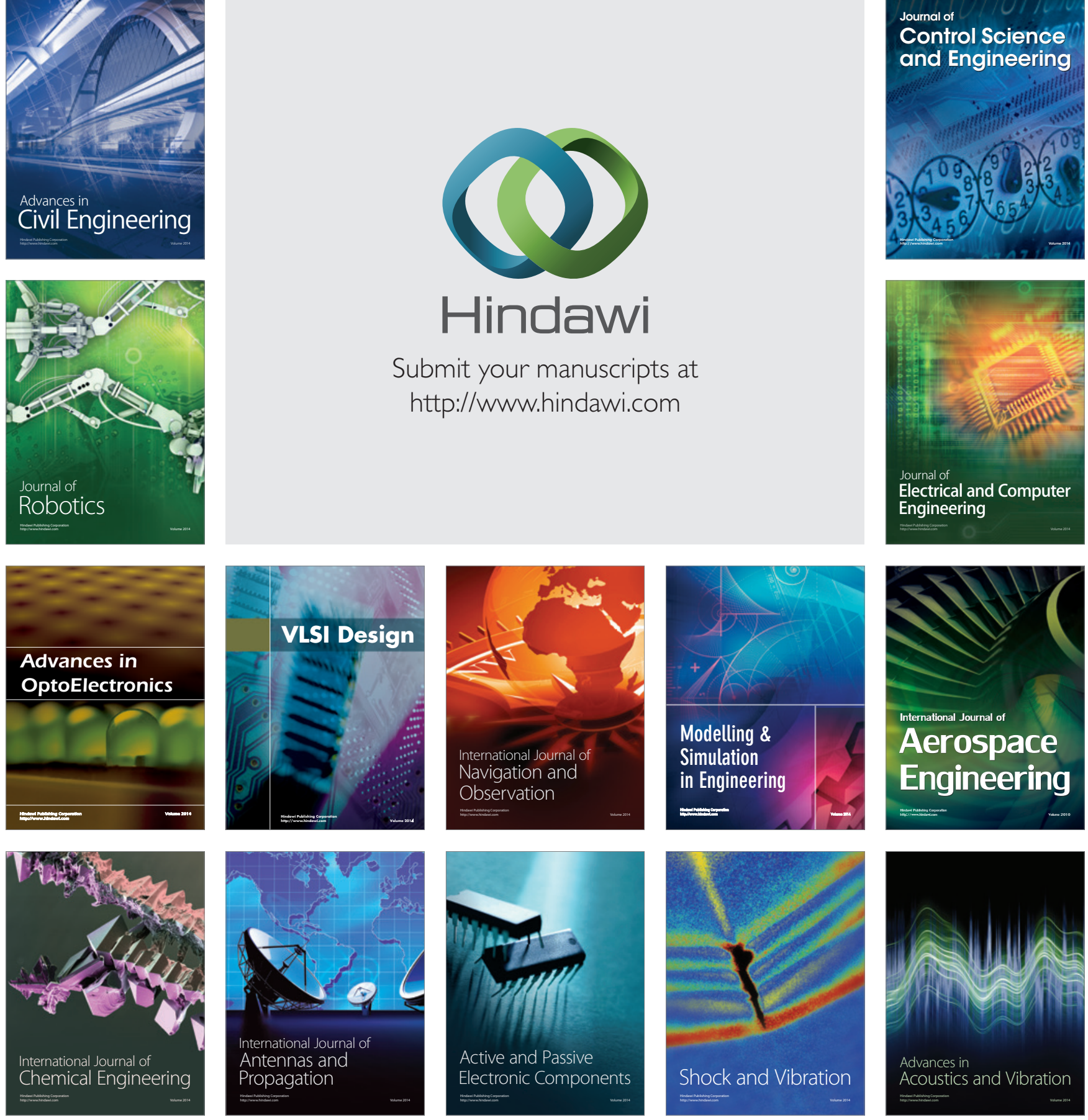